\title{
A Multi-linear CCD Image Correction Method
}

\author{
Hanfei Kuang ${ }^{1}$, Jiexin $\mathrm{Pu}^{2}$, Lei Zhang ${ }^{3}$, Zhonghua Liu ${ }^{4}$, and Bo Peng ${ }^{5}$ \\ 1,2,3,4,5 College of Information Engineering, Henan University of Science and \\ Technology, China \\ 1hanfeikuang@163.com, ${ }^{2}$ pujiexin@126.com, ${ }^{3}$ why219@163.com, \\ ${ }^{4}$ lzhua_217@163.com, ${ }^{5} 185418834 @ q q . c o m$
}

\begin{abstract}
With the aggravation of terrorist activities and frequent occuring of all kinds of car bomb attacks, social and public safety issues have become the focus of attention of the whole world. The vehicle chassis foreign body detection system developed in this paper is mainly used in all kinds of important occasions, to achieve real-time and safe detection of vehicles which is helpful to protect the lives and property of citizens, and to block the invasion and transimission of drugs and other harmful substances. Traditional vehicle chassis detection is carried out by security personnel with dedicated portable underbody detection tool, which is time-consuming, laborious and ineffective. In this paper, we construct a intelligent vehicle chassis image detection system. However, there is always distortion in the real time vehicle chassis image captured by multi-line CCD array which therefore needs to be corrected. This paper presents an image correction method for multi linear CCD. Firstly, Sobel differential operator is used to detect the binarized image of the edge of the vehicle chassis on horizontal and vertical directions. Secondly, we use Radon transform to detect angle of inclination of traveling distorted image captured by line scan cameras based on the results of edge detection, using shear transformation to correct binarized chassis image. Finally, a standard chassis image can be obtained based on image interpolation, the characteristic of Sobel operator and recapture the image. Experimental results show that this proposed method is simple and insensitive of stains and light. In addition, a standard chassis image can be obtained after completing the distortion correction.
\end{abstract}

Keywords: image correction, Sobel algorithm, edge detection, shear transformation

\section{Introduction}

As a convenient means of transportation all around the world, cars has not only brought people convenient transportation but also a threat to public safety such as bombs hidden in vehicle chassis, weapons, chemical dangerous articles, drugs, illegal smuggling, etc. In our country it is always the case that vehicle chassis is applied to hide drugs and dangerous articles, which is harmful to people's life safety and social stability, especially at the airport, gymnasium, nuclear power station, government buildings, embassies, border and military bases and other important circumstances. Therefore, it is necessary to check automobile chassis. However, the traditional detection method based on artificial detection cannot satisfy the requirement of safety testing while auto chassis on-line monitoring based on intelligent image processing technology draws more and more attention.

This paper is based on the vehicle chassis foreign intelligent recognition system, and the data source processed before is almost based on the images captured by plane array ca meras, the advantage of which is that obtaining the two-dimensional image information di rectly and the simplicity of principle of shooting images. And its disadvantages are that th e number of lines is not linear array, the frame rate is easy to be restricted, and the single 
plane array $\mathrm{CCD}[1]$ is difficult to meet the requirements of wide field of view although th e number of pixels is much.And by adopting a buried linear CCD in this paper, the line of pixels has not only a high resolution, but also an accurate location; which can be continuo usly shooting images that is fixed width in motion, and the length of image can be infinitel $y$ extended,besides there is no image tailing phenomenon;it can Figure out the image quic kly and the image information is rich, especially

it can achieve a higher detection accuracy.

But in the image the movement condition of the car is unknown, leading to the condition of unknown image distortion, while the car chassis collected at the scene of the equipment may have foreign body. Therefore, the correction algorithm for the situation of the foreign body should have strong anti-interference.

Many scholars have studied these approximate geometric correction algorithm, such as Yasser[2] and SavopoI[3] who use direct linear transformation model for the SPOT image geometric correction, not separately to solve the interior and exterior orientation elements, with direct transformation of the picpointed coordinate and the corresponding object space for image processing; Jay[4] and Baltsavias[5] who use polynomial correction to line array imaging, which takes the deformation of the remote sensing image as a whole and consider it as a result of all kinds of basic deformation which take effects at the same time. Therefore it construct a mathematical model by employing an appropriate polynomial for the deformation structure so as to avoid the complex process of camera imaging;Vincent[6] and Fraser[7] who have studied the rational function model in the application of image geometric correction and three-dimensional reconstruction the common characteristics of whose algorithms are that the ground control points are set beforehand and the precision of the algorithm is closely related to the precision, distribution range, quantity and correction of ground control points. However, in fact, in the automobile chassis foreign body detection system, we can not set the ground control points in advance for vehicles appear randomly.

So for automobile chassis detection system, the research on the line array CCD camera image geometric correction technology is rarely reported. It is also not available in almost literature research on moving direction of the distortion. In this paper, we can only refer to the relevant methods in plane array cameras to realize the image distortion correction and finally obtain a complete high-quality automobile chassis images.

\section{The General Framwork of the Proposed Method}

After the analysis of marching distortion from the vehicle chassis image, this paper puts forward the algorithm to correct the chassis distortion image and acquire a standard image after recollection. The block diagram of the algorithm is shown in Figure 1:

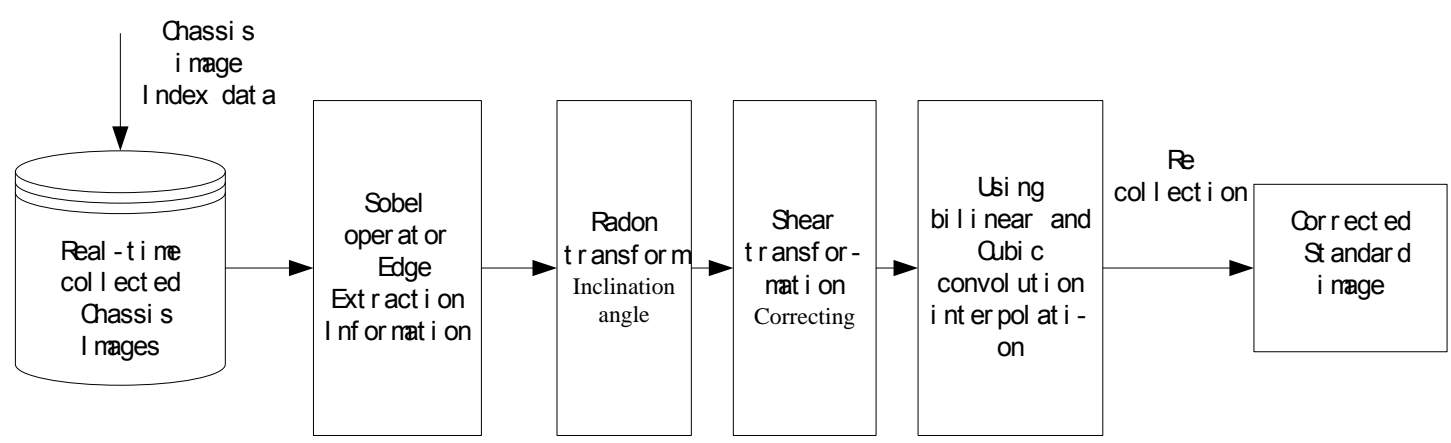

Figure1. Flow Chart of Obtaining the Standard Image 


\section{Edge Detection}

The size of image is the original attribute of an image, the image data we get from the camera is an intuitive response to the natural thing. In the vast majority of cases edge information is used in image processing. Which remove a large number of redundant information from the image while it is also an important attribute of image feature extraction. And moreover, in the vehicle chassis image quoted by this paper, the influence of the light (The sun rays, the internal light source of light is not strictly parallel) is inevitable. To a certain extent edge information can resist the distraction from physical characteristics of the vehicle itself and other factors (collected at different times).

Figure 2(a) is a one dimensional slope edge model of continuous field. When the image range changes from low to high the slope will increase and vice versa. Features including height, angle, horizontal coordinate of the middle point of the inclined plane. When edge height is greater than the threshold set, it is assumed that a boundary exists. As shown in Figure 2(b), when the slope is 90 degrees, it is called the boundary step edge. There is almost no step boundary condition in the real scene image.

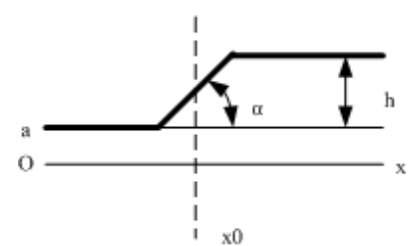

(a)

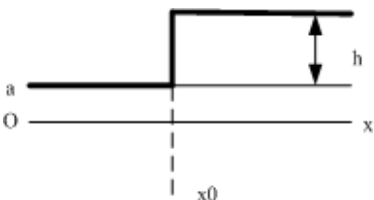

(b)

\section{Figure 2. The Edge of One Dimensional Continuous Domain}

As the points on the image corresponding to the two dimensional plane, a two-dimensional model can be established by extending from one dimensional edge to two-dimensional edge. In the two-dimensional edge model in continuous domain, we believe that the amplitude of discontinuity occurs in the small neighborhood perpendicular to the edge of the side remain the same. Figure 3 is a two-dimensional continuous edge model.

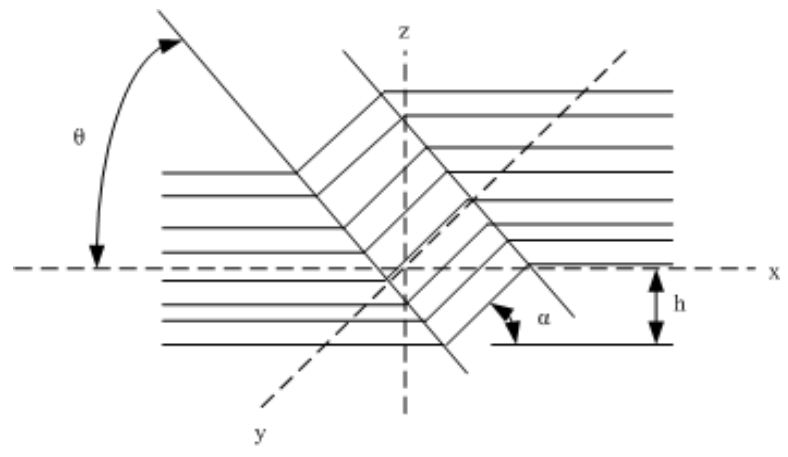

Figure 3. The Edge of a Two-dimensional Continuous Domain

Through the analysis of the theory of edge, this paper implements vehicle chassis distortion correction algorithm on the basis of extracting image edge.

\section{Sobel Algorithm}

Edge is a basic feature of image, and edge detection is often used in image processing, image analysis, pattern recognition, computer vision and human vision. The classical edge detection is based on the detection operator method. The classical edge detection operator method is based on the detection. We investigate the gray level change in a specific 
neighborhood then using the first or second order directional derivative changing rule and detect the edge according to certain criterion common edge detection operators are Prewitt operator, Roberts operator, Laplace operator, Sobel operator[8], Canny operator, LOG operator, etc. This paper adopts the Sobel operator to detect the edge of the binary image in horizontal and vertical detections. Sobel operator means to take a certain pixel as the center and conduct weighted calculation of pixel value to determine whether the point is in extreme state. If the point is in the extreme state, the point is the image edge. This method has the advantages of simple calculation, fast speed.

The implementation is as follows: Using $\mathrm{f}_{\mathrm{x}}(\mathrm{x}, \mathrm{y})$ to represent the pixel value of point $(\mathrm{x}, \mathrm{y}) \cdot \mathrm{f}_{\mathrm{x}}(\mathrm{x}, \mathrm{y})$ is horizontal gradient of point $(\mathrm{x}, \mathrm{y})$ used to detect horizontal edge. The calculation method of $f_{x}(x, y)$ is shown in $(a) . f_{y}(x, y)$ is the vertical direction of gradient of point $(x, y)$ used to detect the vertical edges. The calculation method of $f_{y}(x, y)$ is shown in (b), (c) and (d) is used to detect the edges of the diagonal direction respectively.

\begin{tabular}{|c|c|c|}
\hline-1 & -2 & -1 \\
\hline 0 & 0 & 0 \\
\hline 1 & 2 & 1 \\
\hline
\end{tabular}

(a)Horizontal Direction Operator

\begin{tabular}{|c|c|c|}
\hline 2 & 1 & 0 \\
\hline 1 & 0 & -1 \\
\hline 0 & -1 & -2 \\
\hline
\end{tabular}

(c) $45^{\circ}$ Direction Operator

\begin{tabular}{|l|l|l|}
\hline-1 & 0 & 1 \\
\hline-2 & 0 & 2 \\
\hline-1 & 0 & 1 \\
\hline
\end{tabular}

(b)Vertical Direction Operator

\begin{tabular}{|c|c|c|}
\hline 0 & 1 & 2 \\
\hline-1 & 0 & 1 \\
\hline-2 & -1 & 0 \\
\hline
\end{tabular}

(d) $-45^{\circ}$ Direction Operator

Figure 4. Sobel Algorithm

It needs to set the threshold to determine whether $(\mathrm{x}, \mathrm{y})$ is edge point. We set the average value of the square of the $f_{x}(x, y)$ of each pixel in the image as threshold when detecting horizontal edge. We set the average value of the square of the $f_{y}(x, y)$ of each pixel in the image as threshold when detecting vertical edge. If the threshold value is smaller than $(\mathrm{x}, \mathrm{y})$ then $(\mathrm{x}, \mathrm{y})$ is the edge point.

\subsection{Calculate the Inclination Angle of the Distortion Image}

This paper uses Radon transform to find the inclination angle of automobile chassis distortion image. The basic idea is that the original image is transformed to two parameter spaces by means of linear integral. Radon transform refers to the image projection according to a certain direction [9], The angle corresponding to the distance of the projection in the extreme is the inclination angle of linear array lines. Images stains or uneven illumination will not affect the inclination angle calculated by Radon transform.

The projection formula of two-dimensional Radon transform is (1):

$$
\begin{aligned}
& R\left(x^{\prime}\right)=\int_{-\infty}^{+\infty} f\left(x^{\prime} \cos \theta-y^{\prime} \sin \theta, x^{\prime} \sin \theta-y^{\prime}\right) \\
& \text { where: }\left[\begin{array}{l}
x^{\prime} \\
y^{\prime}
\end{array}\right]=\left[\begin{array}{cc}
\cos \theta & \sin \theta \\
-\sin \theta & \cos \theta
\end{array}\right] \times\left[\begin{array}{l}
x \\
y
\end{array}\right]
\end{aligned}
$$

Because of high imaging frequency and fast speed when the multi linear array CCD collects chassis images, the distortion image can be seen as a rectangular block. The Radon transform of is shown in Figure 4(a) is the coordinate system of the original image of $f(x, y)$ and, the Radon transform. (b) is the linear integral parallel to the y axis. For example, the 2-d line integral of $f(x, y)$ in the vertical direction is the $x$ axis projection of 
the function; the 2-d line integral of $f(x, y)$ in the horizontal direction is the $y$ axis projection of the function. Usually a two-dimensional function $\mathrm{f}(\mathrm{x}, \mathrm{y})$ can project in any angle; the Radon inverse transformation is to reconstruct the original image from the parallel beams projection. When we consider $f(x, y)$ as the image matrix, the Radon transform refers to the projection of a certain angle $\theta$ of the image matrix.

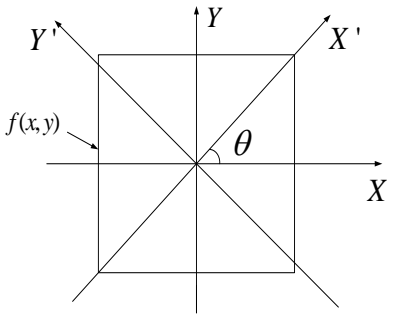

(a) $\mathrm{F}(\mathrm{x}, \mathrm{y})$ of the original image and the Radon transform of the coordinate system

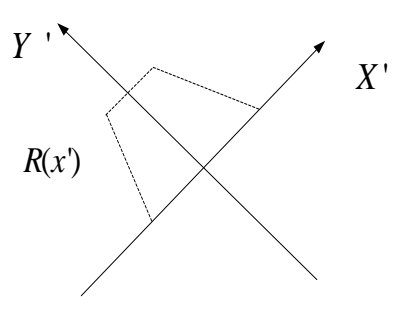

(b) Linear integrals of $\mathrm{F}(\mathrm{x}, \mathrm{y})$ parallel to they $\mathrm{Y}^{\prime}$ axis

\section{Figure 5. Geometric Relationships of Radon Transform}

When the image is tilted, it may be clockwise or counter clockwise. For increasing the robustness of this method, We set the size range of the Radon transform angle of the corrected image as $-90^{\circ} \sim+89^{\circ}$. When get the maximum value of $\mathrm{R}\left(\mathrm{x}^{\prime}\right), \theta$ is maximum inclination angle of the image, the algorithm steps are as follows:

1) Converting the original image to gray image and conducting edge detection, obtaining binary edge image;

2) To Radon transform binary edge image in $-90^{\circ} \sim+89^{\circ}$;

3) To find the maximum value of $\mathrm{R}\left(\mathrm{x}^{\prime}\right)$, namely determining angle $\theta$ when $\mathrm{R}\left(\mathrm{x}^{\prime}\right)$ is the maximum value.

To sum up, using the Radon transform to corrrect the inclination image is through calculating the projection of the image in all directions, looking for the maximum value of the projection direction to determine inclination angle of the image.

\subsection{Shear Transformation Image Correction}

We adopt the inclination angle and shear transformation to correct the binarization image. Shear transformation is a primitive transformation of the affine transformation. In this paper, the shear transformation process is as follows:

$$
\left[\begin{array}{l}
x^{\prime} \\
y^{\prime}
\end{array}\right]=\left[\begin{array}{cc}
1 & -\sin (\alpha \times \pi / 180) \\
-\sin (\beta \times \pi / 180) & 1
\end{array}\right] \times\left[\begin{array}{l}
x \\
y
\end{array}\right]
$$

Among them: $\alpha$ is the horizontal angle of the image and $\beta$ is the vertical angle, $\mathrm{x}, \mathrm{y}$ are horizontal and vertical coordinates in the original image pixels, $x^{\prime}, y$ 'are for pixels after the transformation.

We use bilinear interpolation method in the process of shear transformation, which is described as follows:

The value of the pixel integer coordinate in the original image is floating-point type after a certain transformation, $(i+u, j+v)$ represents coordinate after the transformation, $i, j$ is the integer part, $\mathrm{u}, \mathrm{v}$ is the decimal part. The value of a pixel corresponding to the coordinates after the transformation can be determined by $(i, j)(i+1, j)(i, j+1)$ and $(\mathrm{i}+1, \mathrm{j}+1)$, that is:

$$
\begin{aligned}
& f(i+u, j+v)=(1-u) \times(1-v) \times f(i, j)+(1-u) \times v \times f(i, j+1) \\
& +u \times(1-v) \times f(i+1, j)+u \times v \times f(i+1, j+1)
\end{aligned}
$$

Bilinear interpolation method has large calculation, but has high resized image quality with no discontinuous pixel value. Owing to the low-pass filter nature of the bilinear 
interpolation, the high frequency component is damaged, so the image contour may be blurred to a certain extent.

\section{Image Reacquisition Algorithm}

Including zoom in and out of the image, image scaling is a basic operation in the application digital images, and is widely applied in medical image processing, consumer electronics, and military and other fields. Image zooming is referred to as image resampling, image resolution conversion, scale transformation, etc. Resampling includes an interpolation step, this is a process of generating new pixels with the existing pixels according to a certain algorithm. Existing image scaling algorithm can be roughly divided into traditional interpolation algorithm and edge adaptive algorithm, the former mainly includes the nearest neighbor interpolation, the bilinear interpolation and cubic convolution interpolation method, etc. As they do not consider the image details, that is, the whole image indiscriminate uses the same kind of interpolation function. Therefore, after the scaling the images will be fuzzy or serrate. In this paper, image interpolation is conducted according to the character of the Sobel operator, combined with bilinear and cubic convolution interpolation [10].

\subsection{Adaptive Image Scaling Algorithm Based on Sobel Operator}

Image zooming [11] process is a process of image sampling interpolation, in this paper the algorithm uses bilinear interpolation and cubic convolution interpolation method according to the different local characteristics of the image.

5.1.1. Image Interpolation: Image interpolation[12] is defined as the convolution of a discrete image sampling point and continurous impulse response of two-dimensional reconstruction filter ${ }_{2 \mathrm{D}} \mathrm{h}(\mathrm{x}, \mathrm{y})$.

$$
s(x, y)=\sum_{k} \sum_{l} s(k, l)_{2} h(x-k, y-l)
$$

Among them, the $\mathrm{s}(\mathrm{x}, \mathrm{y})$ is the pixel to be inserted; $\mathrm{s}(\mathrm{k}, \mathrm{l})$ is discrete sampling point; $\mathrm{k}, \mathrm{x}, \mathrm{l}, \mathrm{y}$ are the horizontal and vertical coordinates. To reduce algorithm complexity of two-dimensional kernel interpolation, ${ }_{2 \mathrm{D}} \mathrm{h}(\mathrm{x}, \mathrm{y})$ is divided into the product of impulse response of $\mathrm{x}$ axis and $\mathrm{y}$ axis direction.

$$
{ }_{20} h(x, y)=h(x) h(y)
$$

5.1.2. Bilinear Interpolation and Cubic Convolution Interpolation Method: In bilinear interpolation and cubic convolution interpolation. Interpolation kernel function $\mathrm{h}(\mathrm{x})$ of $\mathrm{x}$ axis and $\mathrm{y}$ axis direction is shown below:

$$
\begin{gathered}
h(x)=\left\{\begin{array}{cc}
1-|x| & 0 \leq x<1 \\
0 & \text { otherwise }
\end{array}\right. \\
h(x)=\left\{\begin{array}{cc}
(A+2)|x|^{3}-(A+3)|x|^{2}+1 & 0<|x|<1 \\
A|x|^{3}-5 A|x|^{2}+8 A|x|-4 a & 1 \leq x \mid<2 \\
0 & 2 \leq|x|
\end{array}\right.
\end{gathered}
$$

Among them, $\mathrm{x}$ is the coordinate distance of pixel point; $\mathrm{A}$ is an adjustable parameter.

From the above we can see that the computational complexity of bilinear interpolation method is lower than the cubic convolution method. From the frequency domain transformation of $\mathrm{H}(\mathrm{X})$ we can see that the bilinear interpolation and cubic convolution method are both low pass filter, in which transitional zone of pass band to stop band of the former is steeper than the latter, and the former has lower high frequency part attenuation and better treatment of high frequency part than the latter. Therefore, the algorithm uses the bilinear interpolation method to complete the pixel interpolation of low frequency part like non edge area. For the high frequency part like image edge details, we 
complete pixel interpolation using cubic convolution interpolation method to guarantee the quality of image zooming.

5.1.3. Description of the Algorithm: This algorithm is roughly divided into three steps:

A Check whether the pixel point of the original image is the edge point;

B To interpolate around the edge points;

C To interpolate around the non-edge points.

According to the known gray levels of pixels in the image, we employ using Sobel operator to detect image edge andcomplete the interpolation of the pixel to be interpolated [12]. Edge detection window is set as window W, using the Sobel operator different direction template to test whether $\mathrm{i}$ is edge point, recording the edge direction. Testing process is as follows:

Calculate convolution value $\mathrm{R}$ of testing template $\mathrm{D}$ and $\mathrm{W}$ of the Sobel operator in every direction:

$$
R=d_{1} w_{1}+d_{2} w_{2}+\cdots+d_{9} w_{9}=\sum_{n=1}^{9} d_{n} w_{n}
$$

Among them, $d_{n}$ and $w_{n}$ are corresponding values of point $n$ of window $W$ and $D$, respectively.

Compare $|R|$ and the threshold $T$ : if $|R|>T$, the point $f$ an edge point, the edge direction is the detecting direction of the template; if $|\mathrm{R}| \leq \mathrm{T}$, the point $\mathrm{f}$ non-edge point. If the point $i$ is an edge point, then interpolation can be conducted based on the edge direction dir , which can be divided into $0^{\circ}, 90^{\circ}, 45^{\circ}$ or $-45^{\circ}$ three cases.

1) If $\operatorname{dir}=0^{\circ}$, interpolation is performed along the horizontal direction;

2) If $\operatorname{dir}=90^{\circ}$, the interpolation is performed in the vertical direction;

3) If $\operatorname{dir}=45^{\circ}$ or $-45^{\circ}$, the interpolation is performed in the diagonal direction.

For a pixel interpolation yet to be interpolated, we use cubic convolution interpolation method to improve the clarity of the target image edge region. To reduce the complexity of the algorithm, bilinear interpolation method is adopted to interpolate them. Let $\mathrm{A}=-1$ in formula (6) then we can get the corresponding filter coefficient $h$. If all $\mathrm{h}$ are $1 / 4$, we can use shift to replace multiplication and division and get gray value of the pixel point to be interpolated. Experimental results show that this method can reduce the complexity and does not affect the image processing effects.

If the point is non-edge point then bilinear interpolation method is adopted to complete the point interpolation to reduce the complexity of the algorithm[13].From left to right, from the top to the bottom traversing all points of the target image, we can complete the interpolation of all pixel points to be interpolated and achieve the zooming of the original image to the target image, then a standard image is acquired.

Three sets of correction distortion images. (a. distortion Figure b. distortion Figure c. standard Figure)
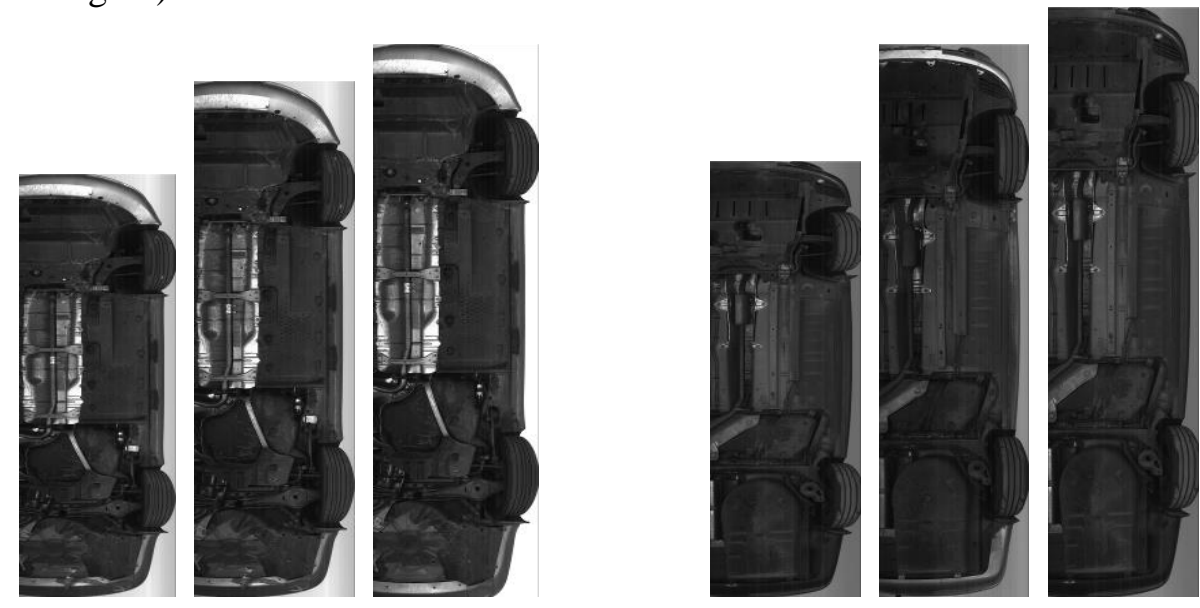
(a)

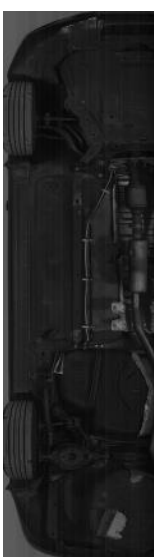

(a) (b)

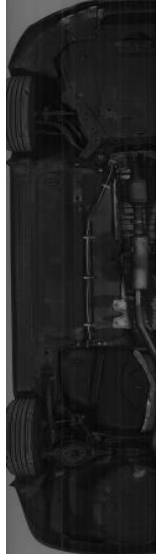

(b) (c)

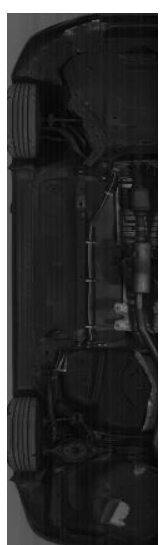

(c)

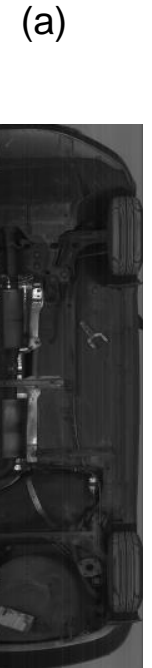

(a) (b)

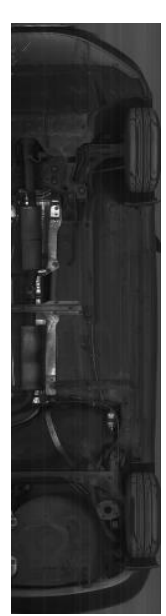

(b) (c)

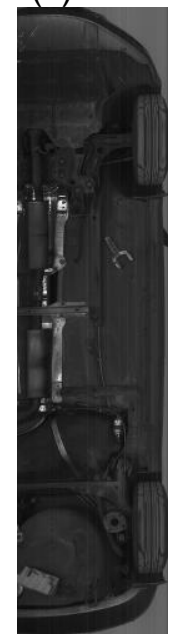

(c)

Figure 6. Experimental Results Figure

\section{Distortion Correction Analysis}

To measure weather the image after distortion correction has satisfactory results, we use the peak signal to noise ratio as analytical standards. PSNR (Peak Signal to Noise Ratio) is an objective criterion of evaluating the image, which is the most common and most widely used evaluation of objective measurement method of quality evaluation, mostly used as an evaluation criterion of signal reconstruction quality in areas such as image compression. PSNR is defined by mean square error (MSE). For two monochrome images $I$ and $K$, if one is a noise approximation to another, then their mean square error is defined as:

$$
M S E=\frac{1}{m n} \sum_{i=0}^{m-1} \sum_{j=0}^{n-1}\|I(i, j)-K(i, j)\|^{2}
$$

Peak signal to noise ratio is defined as:

$$
P S N R=10 \cdot \log _{10}\left(\frac{M A X_{I}^{2}}{M S E}\right)=20 \cdot \log _{10}\left(\frac{M A X_{I}}{\sqrt{M S E}}\right)
$$

In which $\mathrm{MAX}_{\mathrm{I}}$ represents the maximum value of the color of image point, if each sample point is represented by 8 bits, then its value is 255 . More general representation is that if each sample point is represented by a B-bit linear pulse code modulation, then

$$
M A X_{I}=2^{B}-1
$$

Peak signal to noise ratio is typically between $30 \mathrm{~dB}$ to $40 \mathrm{~dB}$, the higher the better. We calculate the PSNR of standard image, initial corrected image and the final image, the resulting value is listed in the following table:

\section{Table1. PSNR Between Resulting Images and The Standard Image}

method

PSNR the traditional method of correcting the image [14] 27.9326 the proposed method 36.1483

From the data in the table it can be seen that the proposed distortion correction algorithm has higher image quality than traditional method, which further validates the superiority of the proposed method. 


\section{Conclusion}

In automotive chassis intelligent foreign object detection system, due to the presence of the vehicle's non uniform and small steering and twist distortion in the linear array CCD image collected, distortion correction is necessary in case of impacting on subsequent image processing. This paper provides a method for correcting the distortion of linear array CCD, which solved this problem that the image quality of the linear array CCD is distorted and the quality of images is worse in the present technical conditions. Firstly, extract edge information of standard image and the image to be corrected with Sobel differential operator, measure inclination angle of distorted image based on the results of edge detection by using Radon transform, and correct binary chassis image combined with the inclination angle through employing shear transform. Finally according to characteristics of Sobel operator, a standard image is generated combined with interpolation worked out by bilinear and cubic convolution method, which means that image is re-captured. After a number of experiments, the experimental results show that the algorithm is feasible and effective, which can effectively solve the problem of linear array $\mathrm{CCD}$ image distortion and improve image quality and can meet real-time requirements.

\section{References}

[1] Y. Cheng, L. Liang and J. Pu, "A geometric correction approach for chasis images with non-uniform speed by linear CCD camera", Journal of IEEE International Conference on Automation and Logistics. (2012), pp. 368-372.

[2] E. M. Yasser and N. Kurt, "Precision Rectification of SPOT Imagery Using the Direct Linear Transformation Model", Journal of Photogrammetric Engineering \& Remote Sensing. vol. 62, no. 1, (1996), pp. 67-72.

[3] F. Savopol and C. Armenakis, "Modeling of IRS-1C Satellite Pan Imagery Using the DLT Approach", Journal of International Archives of Photogrammetry and Remote Sensing. vol. 32, no. 4, (1998), pp. $511-514$.

[4] G. Jay, "Non-Differential GPS as an Alternative Source of Planimetric Control for Rectifying Satellite Imagery", Journal of Photogrammetric Engineering \& Remote Sensing. vol. 67, no. 1, (2001), pp. 49-55.

[5] E. S. Baltsavias, "Metric Information Extraction form SPOT Images and the Role of Polynomial Mapping Functions", Journal of International Archives of Photogrammetry and Remote Sensing. vol. 29, no. B4, (1992), pp. 358-364.

[6] C. T. Vincent and H. Yong, "Updating Solutions of the Rational Function Model Using Additional Control Information ", Journal of Photogrammetric Engineering \& Remote Sensing. vol. 68, no. 7, (2001), pp. 715-724.

[7] C. S. Fraser, E. Baltsavias and A. Gruen, "Processing of Ikonos Imagery for Submeter 3D Positioning and Building Extraction", Journal of Photogrammetry \& Remote Sensing, vol. 56, no. 2, (2002), pp. 177-194.

[8] C. Yuan , Z. Xiong and X. Zhou, "Detection Based on Sobel edge operator ", Journal of Laser and infrared, vol. 39, no. 1, (2009), pp. 85-87.

[9] W. Zhou, "A Comparative Study of the license plate tilt correction algorithm ", Journal of Fujian computer, vol. 26, no. 6, (2010), pp. 65-66.

[10] C. Meijuan, H. Hui and L. Li, "A Fast Edge-oriented Algorithm for Image Interpolation", Journal of Image and Vision Computing, vol. 23, no. 9, (2005), pp. 791-798.

[11] W. Jiang, G. Chen and F. Yang, "Sobel operator based adaptive image scaling algorithm", Journal of Computer Engineering, vol. 36, no. 7, (2010), pp. 214-216.

[12] Y. Zhan, "Adaptive image interpolation algorithm”, Journal of Computer Engineering, vol. 36, no. 16, (2010), pp. 200-202.

[13] K. Ho, S. Mun and L. Aeon. "An Image-scaling Algorithm Using an Area Pixel Model”, Journal of IEEE Transactions on Circuits and Systems for Video Technology, vol. 13, no.6, (2003), pp. 549-553.

[14] X. Zhang, H. Zhu and D. Wang. "A correction method for vehicle chassis image distortion", Academic papers of graduate student in Xi'an University of technology. Xi' an, China, (2013). 


\section{Authors}

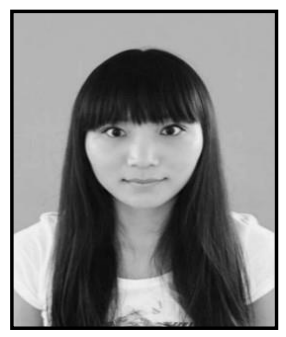

Hanfei Kuang, she was born in October 1991, Henan province, China, M.S student, study in Information Engineering Institute, Henan University of Science and Technology, research interests: image processing, artificial neural nets. 